\title{
PULMONARY THROMBOEMBOLISM DIAGNOSED WITH POINT- OF-CARE ULTRASONOGRAPHY IN THE OPERATING ROOM: A CASE REPORT
}

Y. Fujita ${ }^{1^{*}}$, K. Shimada ${ }^{1}$, K. Nishikawa ${ }^{2}$.

1: Iwaki City Medical Center, Department of Anesthesia, Iwaki, Japan.

2: Fukushima Medical University, Department of Disaster and Comprehensive Medicine,

Fukushima, Japan. *Present affiliation: Kawasaki University of Medical Welfare

\begin{tabular}{lll} 
POD 0 & \multicolumn{1}{c}{ POD 6 } & POD 8 \\
& \\
1st Surgery & Fall in the bathroom & 2nd Surgery \\
Lt.upper arm. & temp. LOS, & ankle fracture \\
& SAH, & unstable anesthesia. \\
& ankle fracture.
\end{tabular}

\section{Case Presentation}

1st surgery

An 84 year-old-woman had left upper arm surgery eight days earlier in this hospital.

\section{A fall on POD 6}

She fell in the bathroom while on the ward during the early morning two days previously. She hit her head and fractured her right ankle. She was unconscious when she was found by a nurse, but soon regained consciousness. Her oxygen saturation was $88 \%$, but increased to $94 \%$ with oxygen via a mask, with a blood pressure of 105/59 $\mathrm{mmHg}$ and heart rate $95 \mathrm{bpm}$. Although subsequent computed tomography (CT) of the head confirmed traumatic subarachnoid hemorrhage, with a small amount of blood in a region of the occipital sulci, no therapeutic intervention was necessary for the head injury. However, she needed surgery for open reduction and internal fixation of the fracture. She had atrial fibrillation, tachycardia of $120 \mathrm{bpm}$, and looked unstable at presentation before induction of anesthesia in the OR, although she responded appropriately to questions.

\section{2nd surgery}

She underwent a non-scheduled surgery because of an ankle fracture. The surgery was performed under general anesthesia using desflurane and air combined with the femoral triangle block and sciatic nerve block through the popliteal approach. The surgery took one hour. Her anesthesia course of about two hours and five minutes was unstable. Her heart rate varied between 95 and $140 \mathrm{bpm}$ and systolic blood pressure varied between 70 and $110 \mathrm{mmHg}$, and frequent iv phenylephrine. Her $\mathrm{SpO}_{2}$ was $92-98 \%$, though $\mathrm{F}_{1} \mathrm{O}_{2}=50 \%$. Her body temperature was $38.5^{\circ} \mathrm{C}$. After removal of iGel, she looked pale, although her $\mathrm{SpO}_{2}$ was $94 \%$ with an oxygen mask at 4 $\mathrm{L} / \mathrm{min}$. Because of the unstable hemodynamic state and unexplained low $\mathrm{SpO}_{2}$ during and after surgery, we performed POC ultrasonography with a portable device to rule out disorders in the heart and lung.

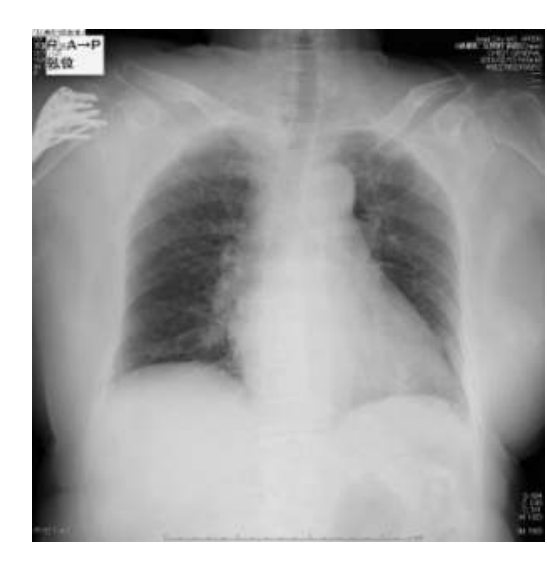
the OR.

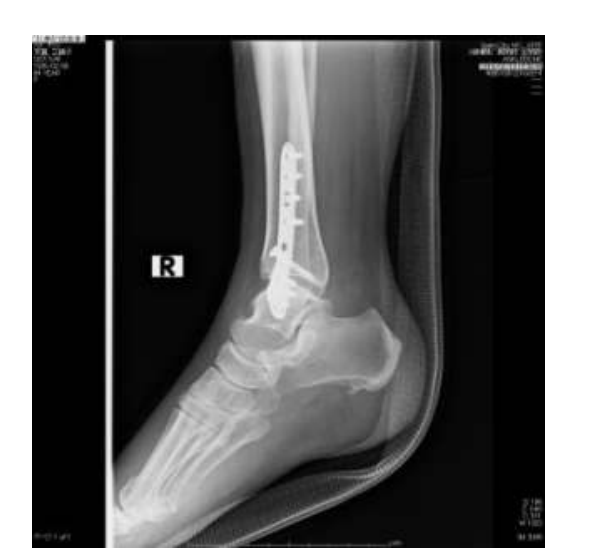

Reference

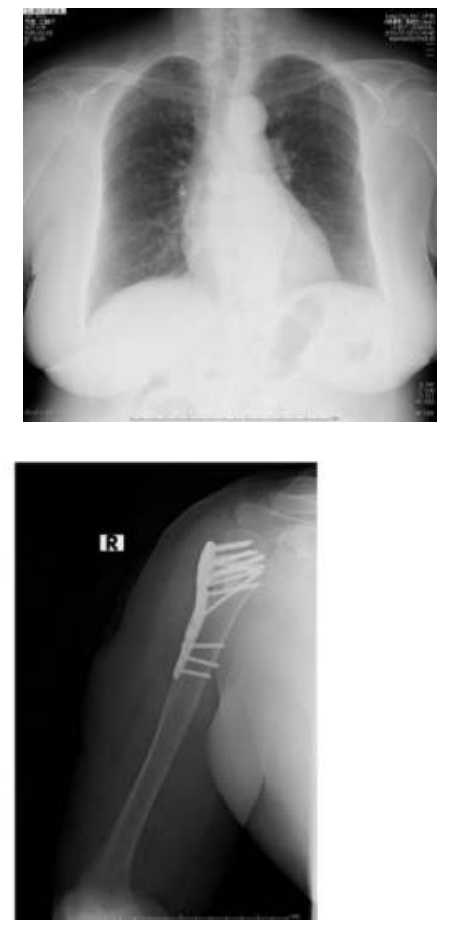

POCUS findings

-Dilated $R V$, reduced free wall motion

-D-shaped LV in PSLA view

- moderate TR with $41 \mathrm{mmHg}$ of TRPG

-Round shape IVC without resp. changes.
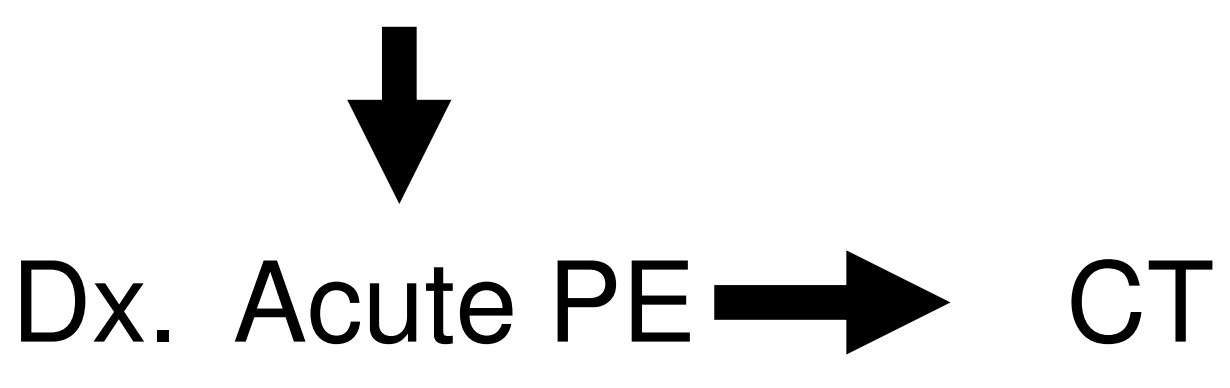

CT angiography

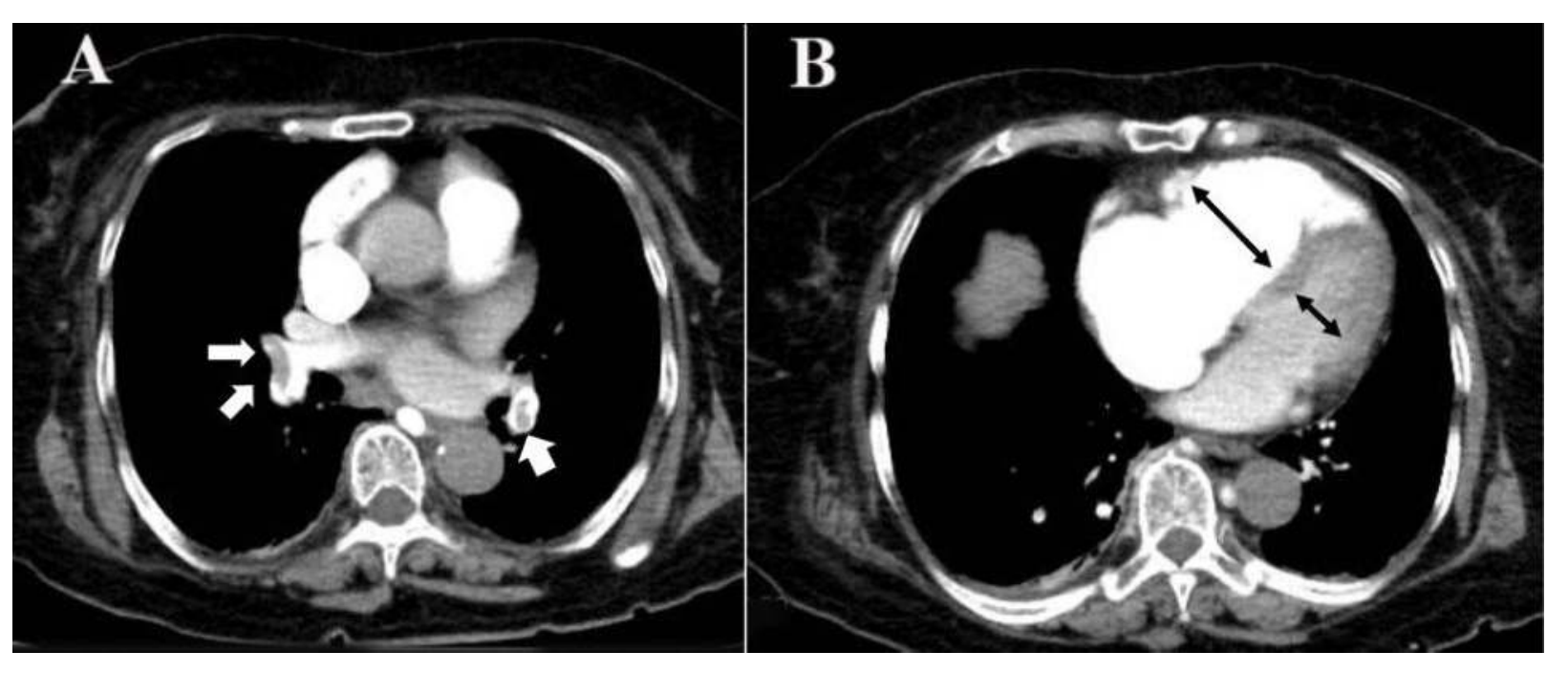

\section{Take home messages}

It was not a simple fall, but fainting!

This case provided two lessons;

1.that PE could manifest as a fall in postoperative patients,

2.that POC ultrasonography is the

first step in the diagnosis of PE in

1. N Engl J Med 2016; 375:1524-31

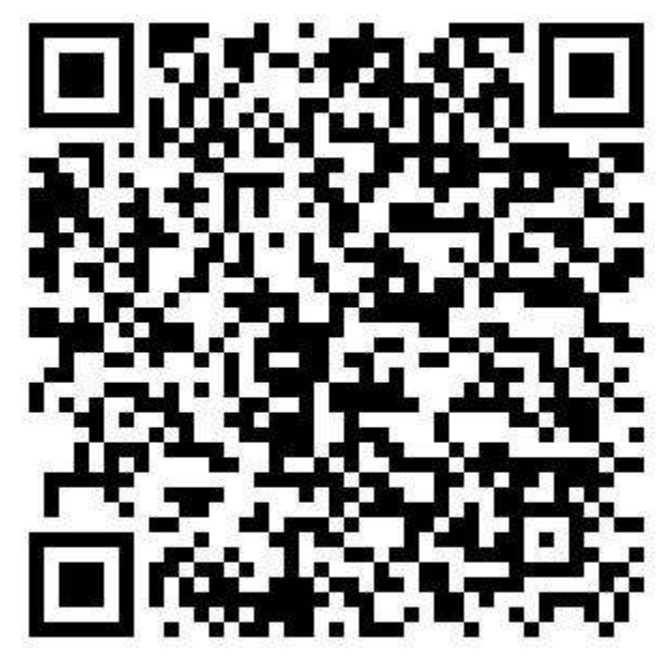

